\title{
ON THE ROBUSTNESS OF PERIODIC SOLUTIONS IN RELAY FEEDBACK SYSTEMS
}

\author{
Mario di Bernardo ${ }^{*}$ Karl Henrik Johansson ${ }^{* *, 1}$ \\ Ulf Jönsson *** Francesco Vasca * \\ * Facoltá di Ingegneria \\ Università del Sannio, Benevento, Italy \\ \{dibernardo|vasca\}@unisannio.it \\ ** Department of Signals, Sensors and Systems \\ Royal Institute of Technology, Stockholm, Sweden \\ kallej@s3.kth.se \\ *** Department of Mathematics \\ Royal Institute of Technology, Stockholm, Sweden \\ ulfj@math.kth.se
}

\begin{abstract}
Structural robustness of limit cycles in relay feedback systems is studied. Motivated by a recent discovery of a novel class of bifurcations in these systems, it is illustrated through numerical simulation that small relay perturbations may change the appearance of closed orbits dramatically. It is shown analytically that certain stable periodic solutions in relay feedback systems are robust to relay perturbations.
\end{abstract}

Keywords: Limit cycles; Sliding Orbits; Perturbation analysis

\section{INTRODUCTION}

Relay feedback systems and, in general, nonsmooth feedback systems tend to self-oscillate (Tsypkin, 1984). Namely, the system evolution tends asymptotically towards stable periodic orbits or limit cycles. Recently, it has been shown that such solutions can undergo abrupt transitions when the system parameters are varied. This led to the discovery of an entirely novel class of bifurcations, involving the interaction between periodic solutions of the system and its discontinuity sets. Despite their widespread use in applications (Flügge-Lotz, 1953; Andronov et al., 1965; Tsypkin, 1984; Åström and Hägglund, 1995; Norsworthy et al., 1997), there are few analytical tools to characterize oscillations in relay feedback systems.

1 Corresponding author.
For example, methods to assess their existence and stability properties are still the subject of much ongoing research (Åström, 1995; Megretski, 1996; Johansson et al., 1997; Johansson et al., 1999; di Bernardo et al., 2000; Georgiou and Smith, 2000; Varigonda and Georgiou, 2001; Gonçalves et al., 2001).

An interesting issue for the considered class of nonsmooth dynamical systems is the robustness properties of the solutions. Due to the discontinuous vector field, classical continuity results for smooth systems are not applicable. Still, it is important in applications to understand if a given solution is robust to unmodeled dynamics, external perturbations, and noise. While there are many results dealing with the robustness of smooth dynamical systems (e.g.,(Wiggins, 1990; Murdock, 1991; Kokotović et al., 1999)), few papers seem to address this issue in the case of systems with nonsmooth vector fields. In the case of relay feed- 
back systems, the available results deal with a quite restrictive class of systems where the transfer function is either close to an integrator (Georgiou and Smith, 2000) or to a second-order nonminimum phase system (Megretski, 1996). Singular perturbations for the smooth part of the system have also been studied (Fridman and Levant, 1996).

In this paper we are interested in the robustness of periodic solutions in relay feedback systems. In particular, we study the case when a system with an ideal relay exhibits an asymptotically stable periodic solution. Then we ask the question if a system with an imperfect implementation of the relay (modeled by a parameter $\varepsilon>0$ ) will also have an asymptotically stable periodic solution. The considered relay implementations include relay with hysteresis, with finite gain (saturation), and with delayed switching. The problem is not trivial, especially, due to the nonsmooth characteristic of the relay. As an illustration, consider the approach, often suggested in the literature, of analyzing relay systems by approximating the relay by a continuous function. There are subtleties when taking the limit as the function tends to the characteristics of the relay. It was recently shown (Johansson et al., 1999) that erroneous results have been derived in the literature when this limit is not dealt with properly. All proofs are presented in (di Bernardo et al., 2002).

The paper is outlined as follows. Relay feedback systems and the perturbations studied in the paper are introduced in Section 2. A motivating example is discussed in Section 3, where it is shown that several interesting bifurcation scenarios appear due to sudden loss of structural stability. Section 4 presents results on perturbations of relay feedback systems. It is shown that if a nominal system exhibits a stable periodic solution, then so will an $\varepsilon$-perturbed system under certain structures of the perturbation. Some concluding remarks and a discussion on future work are presented in Section 5.

\section{RELAY FEEDBACK SYSTEMS}

Consider a nominal relay feedback system

$$
\Sigma_{0}:\left\{\begin{array}{l}
\dot{x}=A x+B u \\
y=C x \\
u=-\operatorname{sgn} y
\end{array}\right.
$$

where $(A, B, C)$ defines a SISO linear time-invariant system of order $n \geq 1$. The relay, defined by the sign function, allows for sliding modes by the setvalued assignment $\operatorname{sgn} 0 \in[-1,1]$ and the interpretation of solutions (trajectories) in the sense of Filippov (Filippov, 1988). A solution $x:[0, \infty) \rightarrow \mathbb{R}^{n}$ of $\Sigma_{0}$ is periodic if there exists a (smallest) period time $T>0$ such that $x(t+T)=x(t)$ for all $t>0$. It is called symmetric if $x(t+T / 2)=-x(t)$ for all $t \geq 0$. The switching plane is defined as $S=\left\{x \in \mathbb{R}^{n}: C x=0\right\}$. A periodic solution $x$ is called simple if the closed orbit $\mathscr{L}=\left\{z \in \mathbb{R}^{n}: \exists t \geq 0, z=x(t)\right\}$ (i) intersects $S$ only twice and (ii) is transversal to $S$ at the intersection points. Note that the condition on transversal intersections is not fulfilled for so called sliding orbits (di Bernardo et al., 2000). The following result gives conditions for existence and stability of periodic solutions (Åström, 1995; Varigonda and Georgiou, 2001). Note that stability refers to exponential stability throughout the paper.

Lemma 2.1. The system $\Sigma_{0}$ has a simple symmetric periodic solution with half-period $t^{*}$ if and only if

$$
\begin{aligned}
f(t) & >0, \quad 0<t<t^{*} \\
f\left(t^{*}\right) & =0, \quad \frac{d f}{d t}(0)>0, \quad \frac{d f}{d t}\left(t^{*}\right)<0,
\end{aligned}
$$

where

$$
\begin{aligned}
f(t) & =C e^{A t} x^{*}-C A^{-1}\left(e^{A t}-I\right) B \\
x^{*} & =\left(e^{A t^{*}}+I\right)^{-1} A^{-1}\left(e^{A t^{*}}-I\right) B .
\end{aligned}
$$

Moreover, it is stable if all eigenvalues of the Jacobian

$$
W=\left(I-\frac{w C}{C w}\right) e^{A t^{*}}, \quad w=\left(e^{A t^{*}}+I\right)^{-1} e^{A t^{*}} B
$$

are in the open unit disc.

Note that the point $x^{*}$ is the intersection point with the switching plane. Extensions of the result are discussed in (Johansson et al., 1997; Johansson et al., 1999; di Bernardo et al., 2000; Varigonda and Georgiou, 2001).

Next we introduce the three alternative relay perturbations that we study in the paper.

(1) A relay feedback system with hysteresis $\varepsilon>0$ is denoted

$$
\Sigma_{\varepsilon}^{H}:\left\{\begin{array}{l}
\dot{x}=A x+B u \\
y=C x \\
u=-\operatorname{sgn}_{\varepsilon}^{H} y,
\end{array}\right.
$$

where the relay is defined as

$$
\begin{aligned}
& u(t)=-\operatorname{sgn}_{\varepsilon}^{H} y(t) \\
& = \begin{cases}-1, & y(t)>\varepsilon \text { or }(-\varepsilon<y(t)<\varepsilon, u(t-)=-1) \\
1, & y(t)<-\varepsilon \text { or }(-\varepsilon<y(t)<\varepsilon, u(t-)=1) .\end{cases}
\end{aligned}
$$

(2) A relay feedback system with the relay replaced by a saturation with steep slope $1 / \varepsilon>0$ is given by

$$
\Sigma_{\varepsilon}^{S}:\left\{\begin{array}{l}
\dot{x}=A x+B u \\
y=C x \\
u=-\operatorname{sgn}_{\varepsilon}^{S} y,
\end{array}\right.
$$

where the relay is defined as

$$
u(t)=-\operatorname{sgn}_{\varepsilon}^{S} y(t)= \begin{cases}-1, & \text { if } y(t)>\varepsilon \\ -y(t) / \varepsilon, & \text { if }-\varepsilon<y(t)<\varepsilon \\ 1, & \text { if } y(t)<-\varepsilon .\end{cases}
$$

(3) A relay feedback system with switching delayed $\varepsilon>0$ amount of time is defined as

$$
\Sigma_{\varepsilon}^{D}:\left\{\begin{array}{l}
\dot{x}=A x+B u \\
y=C x \\
u=-\operatorname{sgn}_{\varepsilon}^{D} y,
\end{array}\right.
$$



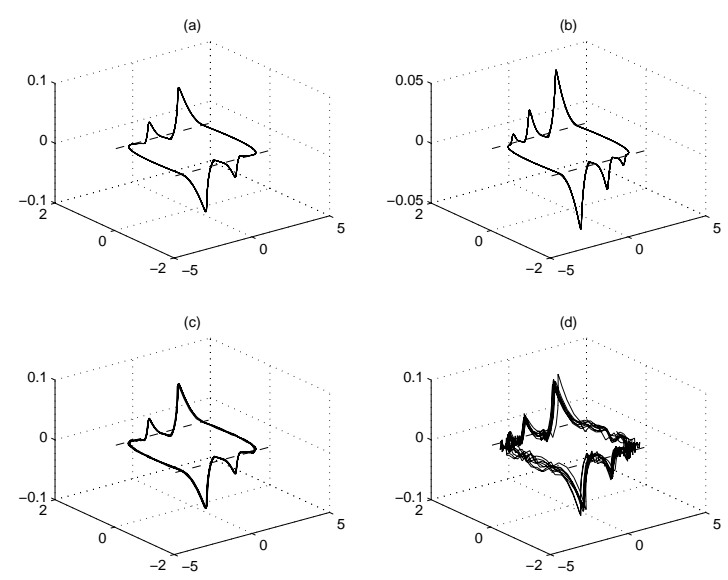

Fig. 1. Oscillations of the third-order relay systems $\Sigma_{0}\left(\mathrm{a}\right.$ and b) and $\Sigma_{\varepsilon}^{H}$ (c and d). The parameter values are $\zeta=0.05, \lambda=\rho=-\sigma=1$ and (a) $\omega=10.3, \varepsilon=0$, (b) $\omega=12, \varepsilon=0$, (c) $\omega=10.3$, $\varepsilon=1 / 1000$, (d) $\omega=10.3, \varepsilon=1 / 100$.

where the relay is simply

$$
u(t)=-\operatorname{sgn}_{\mathcal{\varepsilon}}^{D} y(t)=-\operatorname{sgn} y(t-\varepsilon) .
$$

It should be noticed that the definitions for periodic solutions for $\Sigma_{0}$ directly generalize to the perturbed systems $\Sigma_{\varepsilon}^{D}, \Sigma_{\varepsilon}^{H}$, and $\Sigma_{\varepsilon}^{S}$.

\section{MOTIVATING EXAMPLES}

A third-order relay feedback system recently studied in (di Bernardo et al., 2000; Kowalczyk and di Bernardo, 2001a; Kowalczyk and di Bernardo, 2001b) is now used as a representative example. The linear dynamics is given by

$$
\begin{aligned}
& A=\left(\begin{array}{ccc}
-(2 \zeta \omega+\lambda) & 1 & 0 \\
-\left(2 \zeta \omega \lambda+\omega^{2}\right) & 0 & 1 \\
-\lambda \omega^{2} & 0 & 0
\end{array}\right), \quad B=\left(\begin{array}{c}
1 \\
2 \sigma \rho \\
\rho^{2}
\end{array}\right) \\
& C=\left(\begin{array}{lll}
1 & 0 & 0
\end{array}\right),
\end{aligned}
$$

which corresponds to the transfer function

$$
C(s I-A)^{-1} B=\frac{s^{2}+2 \sigma \rho s+\rho^{2}}{\left(s^{2}+2 \zeta \omega s+\omega^{2}\right)(s+\lambda)} .
$$

This system has been shown to undergo several bifurcation phenomena, which can lead to the occurrence of deterministic chaos (see (Kowalczyk and di Bernardo, 2001b) for a complete description of the bifurcation diagram). This would seem to indicate that periodic solutions of relay systems are sensitive to parameter variations and external disturbances.

In the simplest case, a change in the topology of the solution of $\Sigma_{0}$ can be observed as the parameters are varied. An example is shown in Figs. 1(a)(b), where the transition is depicted from a periodic solution characterized by two segments of sliding motion each half-period to one containing three sections of sliding. More complex scenarios are also possible corresponding to a sudden loss of structural stability. The system can for example exhibit so-called
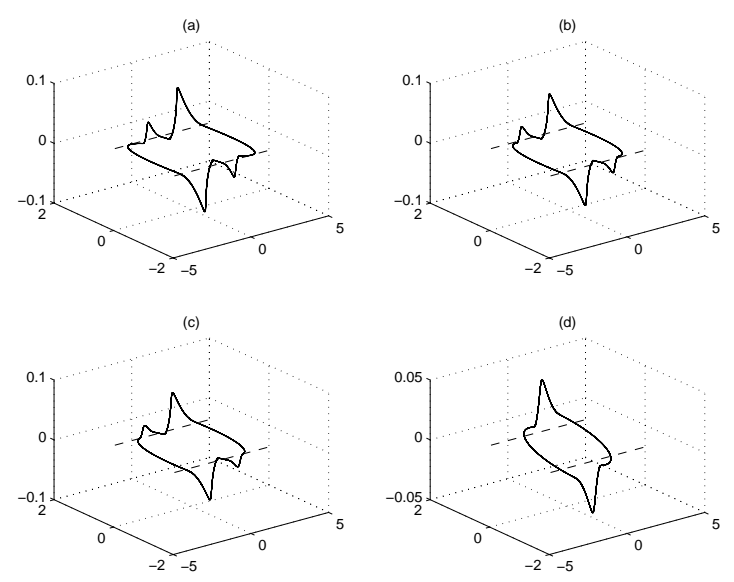

Fig. 2. Oscillations of perturbed third-order relay system $\Sigma_{\varepsilon}^{S}$ with the same parameters as in Fig. 1. The perturbation is (a) $\varepsilon=0$, (b) $\varepsilon=1 / 500$, (c) $\varepsilon=1 / 250$, (d) $\varepsilon=1 / 100$.

period-doubling cascades to chaos (Kowalczyk and di Bernardo, 2001b) or in some cases an abrupt transition from regular to chaotic motion (Verghese and Banerjee, 2001). The occurrence of these phenomena has been recently explained in the literature as due to the occurrence of new bifurcations, unique to nonsmooth systems. The formation of periodic solutions with sliding (or sliding orbits), for example, has been explained by identifying so-called sliding bifurcations (di Bernardo et al., 2000). These are due to interactions between periodic orbits of the system and regions on the discontinuity set where sliding is possible. The existence of unexpected transitions involving self-oscillations of relay feedback systems motivates the study of how persistent periodic solutions are. We restrict our attention to the effects of perturbations to the relay characteristics. Our numerics seem to suggest that oscillations in relay feedback systems are unexpectedly robust to perturbations of the relay characteristic. Fig. 1(c) shows, for instance, that the orbit characterized by two sections of sliding motion for $\Sigma_{0}$ depicted in Fig. 1(a) is robust to a small hysteresis $\left(\Sigma_{\varepsilon}^{H}\right.$ with $\varepsilon=1 / 1000$ ). We see, though, that as the perturbation is increased the effects of the hysteresis cannot be neglected (Fig. 1(d)). Nevertheless, the influence of the underlying unperturbed orbit remains clearly visible.

Similar effects as in Fig. 1 are shown in Fig. 2 but for $\Sigma_{\varepsilon}^{S}$, in which case the system is perturbed by substituting the relay element with a finite gain saturation. Again we see that for relatively small value of the perturbation (high value of the gain), the perturbed orbits (Fig. 2(b)-(c)) stay close to the unperturbed one (Fig. 2(a)). Lower values of the gain though cause the transition to the different orbit depicted in Fig. 2(d). Note that the persistence observed in the system is quite remarkable. Substituting the relay with a saturation prevents the occurrence of sliding mode without causing a destruction of the unperturbed solution structure. This structural robustness is also observed in the case 

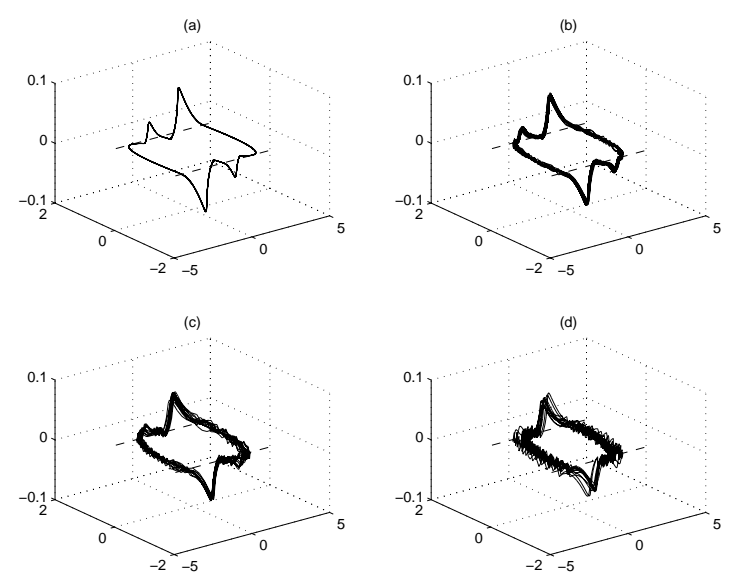

Fig. 3. Oscillations of the delayed relay system $\Sigma_{\varepsilon}^{D}$ for the same parameter values as in Fig. 1. (a) $\varepsilon=0$, (b) $\varepsilon=1 / 200$, (c) $\varepsilon=1 / 125$, (d) $\varepsilon=1 / 100$.
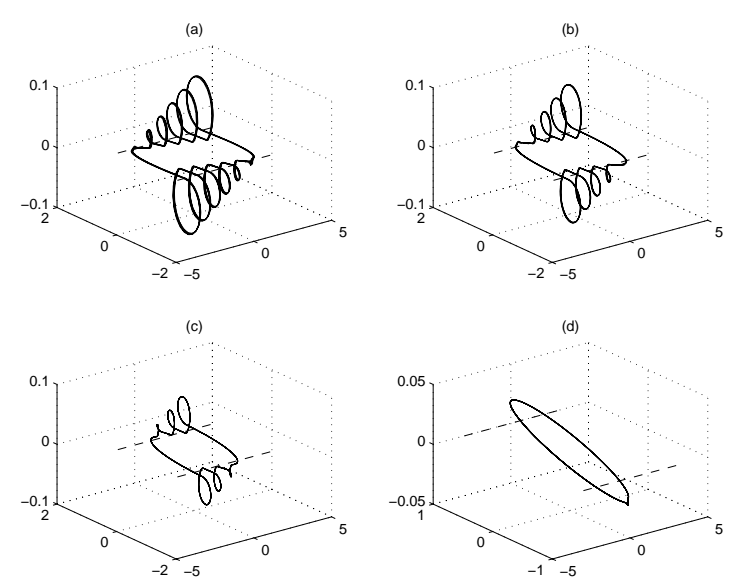

(d)

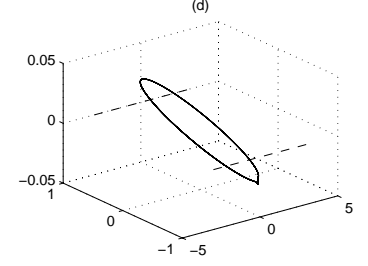

Fig. 4. Oscillations of perturbed third-order relay system $\Sigma_{\varepsilon}^{S}$ with parameters $\zeta=-0.07, \lambda=$ $0.05, \rho=-\sigma=1$ and $\omega=10$. (a) $\varepsilon=0$, (b) $\varepsilon=1 / 200$, (c) $\varepsilon=1 / 100$, (d) $\varepsilon=1 / 50$.

of $\Sigma_{\varepsilon}^{D}$, where the relay is perturbed by adding a small delay. Fig. 3 shows how the periodic orbit under investigation varies as the delay is increased. Despite the onset of high-frequency oscillations, the structure of the unperturbed orbit is still preserved. More dramatic effects are observed when the robustness of a more complex dynamical behavior is investigated. When the chaotic attractor shown in Fig. 4(a) is perturbed by substitution of the relay with a high gain saturation, its topology changes to the one shown in Fig. 4(b) (characterized by a lower number of lobes). Further variation of the gain, causes a further reduction of the lobes (Fig. 4(c)) followed by the appearance of a stable asymmetric periodic solution (Fig. 4(d)). The effects of a small hysteresis on the same chaotic attractor are even more evident as shown in Fig. 5, where $\Sigma_{\varepsilon}^{H}$. Here we see the attractor structure changing rapidly as the perturbation is increased.

The simulations reported above highlight the need for appropriate theoretical tools to systematically carry out the robustness analysis of oscillations in relay systems. In what follows, perturbation analysis of so-
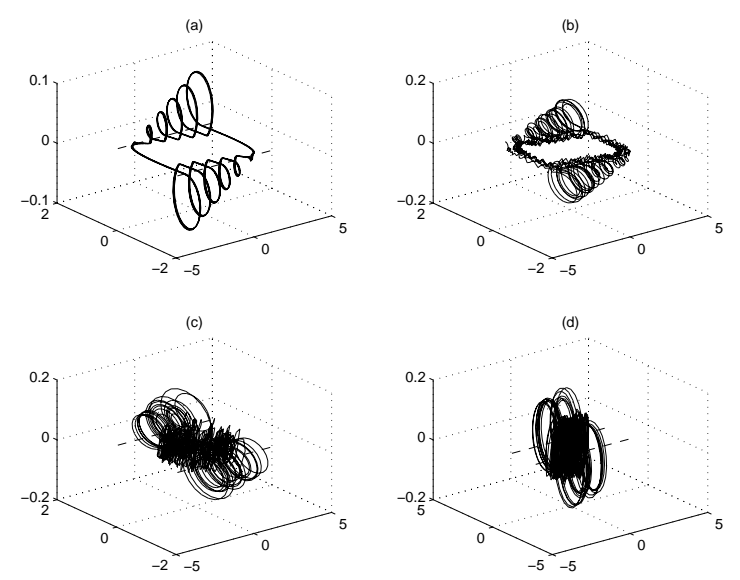

Fig. 5. Oscillations of the hysteresis perturbed relay system $\Sigma_{\varepsilon}^{H}$ for the same parameter values as in Fig. 4, but with (a) $\varepsilon=0$, (b) $\varepsilon=1 / 100$, (c) $\varepsilon=1 / 20$, (d) $\varepsilon=1 / 10$.

called simple periodic solutions is discussed. These intersect the switching plane transversally, which make them easier to analyze using classical Poincaré techniques. Note that it seems that tangential intersections plays an important role in some of the bifurcation phenomena illustrated above, cf., bifurcation analysis in (di Bernardo et al., 2000). The robustness analysis of periodic solution that hits or leaves the switching plan tangentially will be studied in future work.

\section{PERTURBATION ANALYSIS}

In this section we study different perturbations of the nominal relay feedback system $\Sigma_{0}$. Given some rather non-restrictive assumptions, we will see that a stable periodic solution of $\Sigma_{0}$ is persistent, in the sense that the perturbed system $\Sigma_{\varepsilon}^{P}$ also has a stable periodic solution regardless of the perturbation $P \in\{H, S, D\}$. The following theorem summarizes the result of the section.

Theorem 4.1. Suppose the relay feedback system $\Sigma_{0}$ has a simple symmetric periodic solution with a strictly stable Jacobian (as defined in Lemma 2.1). Then, there exists $\varepsilon_{0}>0$ such that for each $\varepsilon \in\left(0, \varepsilon_{0}\right)$ the perturbed relay feedback systems $\Sigma_{\varepsilon}^{H}, \Sigma_{\varepsilon}^{S}$, and $\Sigma_{\varepsilon}^{D}$ all have simple symmetric stable periodic solutions.

The proof uses three lemmas which state sufficient conditions for $\Sigma_{\varepsilon}^{H}, \Sigma_{\varepsilon}^{S}$, and $\Sigma_{\varepsilon}^{D}$, respectively, to exhibit periodic solutions. The lemmas, which are presented next, are derived using techniques from the recent literature on relay feedback systems, e.g., (Åström, 1995). The main contribution of Theorem 4.1 is however on how to connect the existence of a periodic solution for $\Sigma_{0}$ with the existence of a periodic solution for a perturbed system. It turns out that it is easy to make the connection for $\Sigma_{\varepsilon}^{H}$ and $\Sigma_{\varepsilon}^{D}$, while $\Sigma_{\varepsilon}^{S}$ requires some more analysis. The proofs are presented in (di Bernardo et al., 2002). 
Throughout the section, we make the following standing assumption.

Assumption 4.1. The relay feedback system $\Sigma_{0}$ has a simple symmetric periodic solution with half-period $t^{*}$. All eigenvalues of $W$ (defined in Lemma 2.1) are inside the unit disk.

Consider the relay feedback system with hysteresis $\Sigma_{\varepsilon}^{H}$. We note that with a straightforward modification of Lemma 2.1 the following result holds, cf., ( $⿱$ Aström, 1995).

Lemma 4.1. The system $\Sigma_{\varepsilon}^{H}$ has a stable simple symmetric periodic solution with half-period $\tau$ if (i)

$$
\begin{aligned}
f^{H}(t, \varepsilon) & >0, \quad 0<t<\tau \\
f^{H}(\tau, \varepsilon) & =0, \quad \frac{d f^{H}}{d t}(0, \varepsilon)>0, \quad \frac{d f^{H}}{d t}(\tau, \varepsilon)<0
\end{aligned}
$$

where

$$
\begin{aligned}
f^{H}(t, \varepsilon) & =C e^{A t} z^{*}-C A^{-1}\left(e^{A t}-I\right) B+\varepsilon \\
z^{*} & =\left(e^{A \tau}+I\right)^{-1} A^{-1}\left(e^{A \tau}-I\right) B
\end{aligned}
$$

and (ii) all eigenvalues of the Jacobian

$$
\begin{aligned}
W^{H}(\varepsilon) & =\left(I-\frac{w^{H} C}{C w^{H}}\right) e^{A \tau} \\
w^{H}(\varepsilon) & =\left(e^{A \tau}+I\right)^{-1} e^{A \tau} B
\end{aligned}
$$

are in the open unit disc.

If the nominal system $\Sigma_{0}$ generates a closed orbit as specified in Lemma 2.1, one can now show that also $\Sigma_{\varepsilon}^{H}$ generates one if $\varepsilon>0$ is small using the Implicit Function Theorem.

Consider the perturbed relay feedback system $\Sigma_{\varepsilon}^{S}$, where the relay is replaced by a saturation with steep slope. Introduce the notation $\phi_{+}$for the flow of $\dot{x}=$ $A x+B, \phi_{-}$for the flow of $\dot{x}=A x-B$, and $\phi_{\varepsilon}$ for the flow of $\dot{x}=(A-B C / \varepsilon) x$. The following result similar to Lemma 2.1 then holds.

Lemma 4.2. The system $\Sigma_{\varepsilon}^{S}$ has a stable simple symmetric periodic solution with half-period $\tau_{1}+\tau_{2}+\tau_{3}$ if (i)

$$
\begin{array}{cc}
0<f_{1}^{S}(t, \varepsilon)<\varepsilon, & 0<t<\tau_{1} \\
\varepsilon<f_{2}^{S}(t, \varepsilon), & 0<t<\tau_{2} \\
0<f_{3}^{S}(t, \varepsilon)<\varepsilon, & 0<t<\tau_{3} \\
f_{1}^{S}\left(\tau_{1}, \varepsilon\right)=\varepsilon, \quad f_{2}^{S}\left(\tau_{2}, \varepsilon\right)=\varepsilon, & f_{3}^{S}\left(\tau_{3}, \varepsilon\right)=0 \\
\frac{d f_{1}^{S}}{d t}(0, \varepsilon)>0, & \frac{d f_{1}^{S}}{d t}\left(\tau_{1}, \varepsilon\right)>0 \\
\frac{d f_{2}^{S}}{d t}(0, \varepsilon)>0, & \frac{d f_{2}^{S}}{d t}\left(\tau_{2}, \varepsilon\right)<0 \\
\frac{d f_{3}^{S}}{d t}(0, \varepsilon)<0, & \frac{d f_{3}^{S}}{d t}\left(\tau_{3}, \varepsilon\right)<0
\end{array}
$$

where

$$
\begin{aligned}
f_{1}^{S}(t, \varepsilon) & =C \phi_{\varepsilon}\left(t, z^{*}\right), f_{2}^{S}(t, \varepsilon)=C \phi_{-}\left(t, z_{1}^{*}\right) \\
f_{3}^{S}(t, \varepsilon) & =C \phi_{\varepsilon}\left(t, z_{2}^{*}\right) \\
z^{*} & =-\phi_{\varepsilon}\left(\tau_{3}, z_{2}^{*}\right), z_{1}^{*}=\phi_{\varepsilon}\left(\tau_{1}, z^{*}\right) \\
z_{2}^{*} & =\phi_{-}\left(\tau_{2}, z_{1}^{*}\right),
\end{aligned}
$$

and (ii) all eigenvalues of the Jacobian

$$
W^{S}(\varepsilon)=W_{3}^{S}(\varepsilon) W_{2}^{S}(\varepsilon) W_{1}^{S}(\varepsilon)
$$

are in the open unit disc with

$$
\begin{aligned}
W_{1}^{S}(\varepsilon) & =\left(I-\frac{M_{1} A z^{*} C}{C M_{1} A z^{*}}\right) M_{1} \\
W_{2}^{S}(\varepsilon) & =\left(I-\frac{w^{S} C}{C w^{S}}\right) e^{A \tau_{2}} \\
W_{3}^{S}(\varepsilon) & =\left(I-\frac{M_{3} A z_{2}^{*} C}{C M_{3} A z_{2}^{*}}\right) M_{3} \\
M_{1} & =e^{A \tau_{1}}, M_{3}=e^{A \tau_{3}} \\
w^{S}(\varepsilon) & =e^{A \tau_{2}}\left(A z_{1}^{*}-B\right) .
\end{aligned}
$$

The robustness result can now be shown also for $\Sigma_{\varepsilon}^{S}$, where the proof is based on a contraction mapping argument together with that $W^{S}(\varepsilon)$ will be close to $W$ for small $\varepsilon$.

Consider the perturbed relay feedback system $\Sigma_{\varepsilon}^{D}$, where the switching is delayed a short amount of time.

Lemma 4.3. The system $\Sigma_{\varepsilon}^{D}$ has a stable simple symmetric periodic solution with half-period $\tau+\varepsilon$ if (i)

$$
\begin{array}{rlrl}
f_{1}^{D}(t, \varepsilon) & >0, & 0<t<\varepsilon \\
f_{2}^{D}(t, \varepsilon) & >0, & 0<t<\tau \\
f_{2}^{D}(\tau, \varepsilon) & =0, & \frac{d f_{1}^{D}}{d t}(0, \varepsilon)>0, & \frac{d f_{2}^{D}}{d t}(\tau, \varepsilon)<0,
\end{array}
$$

where

$$
\begin{aligned}
& f_{1}^{D}(t, \varepsilon)=C \phi_{+}\left(t, z^{*}\right) \\
& f_{2}^{D}(t, \varepsilon)=C \phi_{-}\left(t, \phi_{+}\left(\varepsilon, z^{*}\right)\right) \\
z^{*}= & \left(e^{A(\tau+\varepsilon)}+I\right)^{-1} A^{-1}\left(2 e^{A \tau}-e^{A(\tau+\varepsilon)}-I\right) B,
\end{aligned}
$$

and (ii) all eigenvalues of the Jacobian

$$
W^{D}(\varepsilon)=W_{2}^{D}(\varepsilon) W_{1}^{D}(\varepsilon)
$$

are in the open unit disc with

$$
\begin{aligned}
W_{1}^{D}(\varepsilon) & =e^{A \varepsilon}, \quad W_{2}^{D}(\varepsilon)=\left(I-\frac{w^{D} C}{C w^{D}}\right) e^{A \tau} \\
w^{D}(\varepsilon) & =e^{A \tau}\left(A \phi_{+}\left(\varepsilon, z^{*}\right)-B\right) .
\end{aligned}
$$

The robustness result follows similarly to the proof for $\Sigma_{\varepsilon}^{S}$, but with application of Lemma 4.3.

\section{CONCLUSIONS AND FUTURE WORK}

Perturbation analysis in relay feedback systems was discussed. It was shown that stable simple symmetric 
periodic solutions are persistent under small variations in the relay characteristic. Simulations showed that if the orbits are not simple (i.e., do not intersect the switching plane transversally twice per period), then sensitive solutions may appear. Examples of this include so-called sliding orbits. Future work include studying perturbations of sliding orbits in detail. Bifurcation phenomena can cause a sudden loss of structural stability, which recently was analytically investigated (di Bernardo et al., n.d.).

The robustness result in this paper can be extended to more general piecewise affine systems. It is interesting to consider relay feedback systems with other imperfections, such as model errors in the linear dynamics and unmodeled dynamics. It is straightforward to extend Theorem 4.1 to a class of system $\Sigma_{\varepsilon}^{M}$, which has an ideal relay but the linear system is replaced by smooth functions $A(\varepsilon), B(\varepsilon), C(\varepsilon)$, such that $(A(0), B(0), C(0))=(A, B, C)$.

\section{REFERENCES}

Andronov, A. A., S. E. Khaikin and A. A. Vitt (1965). Theory of Oscillators. Pergamon Press. Oxford.

Åström, K. J. (1995). Oscillations in systems with relay feedback. In: Adaptive Control, Filtering, and Signal Processing (K. J. Åström, G. C. Goodwin and P. R. Kumar, Eds.). Vol. 74 of IMA Volumes in Mathematics and its Applications. pp. 1-25. Springer-Verlag.

Åström, K. J. and T. Hägglund (1995). PID Controllers: Theory, Design, and Tuning. second ed.. Instrument Society of America. Research Triangle Park, NC.

di Bernardo, M., K. H. Johansson and F. Vasca (2000). Self-oscillations and sliding in relay feedback systems: Symmetry and bifurcations. International Journal of Bifurcations and Chaos 11(4), 1121-1140.

di Bernardo, M., K. H. Johansson, U. Jönsson and F. Vasca (2002). On the robustness of periodic solutions in relay feedback systems. Technical Report TRITA/MAT-02-OS06. Department of Mathematics, Royal Institute of Technology. Stockholm, Sweden.

di Bernardo, M., P. Kowalczyk and A. Nordmark (n.d.). Explaining the onset of complex behaviour in dry-friction oscillators. Submitted.

Filippov, A. F. (1988). Differential Equations with Discontinuous Righthand Sides. Kluwer Academic Publishers.

Flügge-Lotz, I. (1953). Discontinuous Automatic Control. Princeton University Press.

Fridman, L. M. and A. Levant (1996). Higher order sliding modes as a natural phenomenon in control theory. In: Robust Control via Variable Structure \& Lyapunov Techniques (F. Garofalo and L. Glielmo, Eds.). Vol. 217 of Lecture notes in control and information science. pp. 107-133. Springer-Verlag.
Gelig, A. Kh. and A. N. Churilov (1993). Oscillations and Stability of Nonlinear Impulsive Systems. St.Petersburg State Univ., St. Petersburg. (Russian).

Georgiou, T. T. and M. C. Smith (2000). Robustness of a relaxation oscillator. International Journal of Nonlinear and Robust Control 10, 1005-1024.

Gonçalves, J. M., A. Megretski and M. A. Dahleh (2001). Global stability of relay feedback systems. IEEE Trans. on Automatic Control 46(4), 550-562.

Johansson, K. H., A. Barabanov and K. J. Åström (1997). Limit cycles with chattering in relay feedback systems. In: Proc. 36th IEEE Conference on Decision and Control. San Diego, CA. To appear in IEEE Trans. on Automatic Control.

Johansson, K. H., A. Rantzer and K. J. Åström (1999). Fast switches in relay feedback systems. Automatica 35(4), 539-552.

Kokotović, P., H. K. Khalil and J. O’Reilly (1999). Singular Perturbation Methods in Control: Analysis and Design. Classics in Applied Mathematics. SIAM.

Kowalczyk, P. and M. di Bernardo (2001a). On a novel class of bifurcations in hybrid dynamical systems: the case of relay feedback systems. In: Hybrid Systems: Computation and Control (M. di Benedetto and A. L. SangiovanniVincentelli, Eds.). Vol. 2034 of Lecture Notes in Computer Science. Springer-Verlag, New York.

Kowalczyk, P. and M. di Bernardo (2001b). On the existence of stable asymmetric limit cycles and chaos in unforced symmetric relay feedback systems. In: European Control Conference. Porto, Portugal.

Megretski, A. (1996). Global stability of oscillations induced by a relay feedback. In: Preprints IFAC 13th World Congress. Vol. E. San Francisco, CA. pp. 49-54.

Murdock, J. A. (1991). Perturbations: Theory and Methods. John Wiley \& Sons.

Norsworthy, S. R., R. Schreier and G. C. Temes (1997). Delta-Sigma Data Converters-Theory, Design, and Simulation. IEEE Press. New York.

Tsypkin, Ya. Z. (1984). Relay Control Systems. Cambridge University Press. Cambridge, U.K.

Varigonda, S. and T. T. Georgiou (2001). Dynamics of relay relaxation oscillators. IEEE Trans. on Automatic Control 46(1), 65-77.

Verghese, G. and Banerjee, S., Eds.) (2001). Nonlinear Phenomena in Power Electronics. IEEE Press.

Wiggins, S. (1990). Introduction to Applied Nonlinear Dynamical Systems and Chaos. Springer-Verlag. 